\title{
Exploring the molecular pathogenesis associated with T-cell prolymphocytic leukemia based on a comprehensive bioinformatics analysis
}

\author{
ZHANGZHEN SHI ${ }^{1}$, JING YU ${ }^{2}$, HUI SHAO ${ }^{1}$, KAILIANG CHENG ${ }^{3}$, JINGJIE ZHAI ${ }^{4}$, QI JIANG ${ }^{2}$ and HONGJUN LI $^{5}$ \\ Departments of ${ }^{1}$ Hematology and Oncology, ${ }^{2}$ Nephrology and ${ }^{3}$ Radiology, China-Japan Union Hospital of Jilin University, \\ Changchun, Jilin 130033; ${ }^{4}$ Department of Implantology, Jilin University Stomatology Hospital, Changchun, Jilin 130021; \\ ${ }^{5}$ Medical Examination Center, China-Japan Union Hospital of Jilin University, Changchun, Jilin 130033, P.R. China
}

Received February 14, 2016; Accepted February 27, 2017

DOI: $10.3892 /$ ol.2018.8615

\begin{abstract}
As a rare hematological malignancy, T-cell prolymphocytic leukemia (T-PLL) has a high mortality rate. However, the comprehensive mechanisms of the underlying pathogenesis of T-PLL are unknown. The purpose of the present study was to investigate the pathogenesis of T-PLL based on a comprehensive bioinformatics analysis. The differentially expressed genes (DEGs) between T-PLL blood cell samples and normal peripheral blood cell samples were investigated using the GSE5788 Affymetrix microarray data from the Gene Expression Omnibus database. To investigate the functional changes associated with tumor progression, Gene Ontology and Kyoto Encyclopedia of Genes and Genomes pathway enrichment analyses were used on the identified DEGs, followed by protein-protein interaction (PPI) and sub-PPI analysis. Transcription factors and tumor-associated genes (TAGs) were investigated further. The results identified 84 upregulated genes and 354 downregulated genes in T-PLL samples when compared with healthy samples. These DEGs featured in various functions including cell death and various pathways including apoptosis. The functional analysis of DEGs revealed 17 dysregulated transcription factors and 37 dysregulated TAGs. Furthermore, the PPI network analysis based on node degree (a network topology attribute) identified 61 genes, including the core downregulated gene of the sub-PPI network, signal transducer and activator of transcription 3 (STAT3; degree, 13) and the core upregulated gene, insulin receptor substrate-1 (IRS1; degree, 5), that may have important associations with the progression of T-PLL. Alterations to cell functions, including cell death, and pathways, including
\end{abstract}

Correspondence to: Professor Hongjun Li, Medical Examination Center, China-Japan Union Hospital of Jilin University, 126 Xiantai Street, Changchun, Jilin 130033, P.R. China

E-mail: lihongjun1960@126.com

Key words: T-cell prolymphocytic leukemia, differentially expressed genes, pathways, protein-protein interaction, transcription factor apoptosis, may contribute to the process of T-PLL. Candidate genes identified in the present study, including STAT3 and IRSI, should be targets for additional studies.

\section{Introduction}

T-cell prolymphocytic leukemia (T-PLL) has an aggressive course and poor response to conventional therapy, with median survival times ranging between 7 and 30 months (1-4). Although chemotherapeutic drugs, including alemtuzumab and analogues, have significantly improved survival and response rates (5), the survival rate remains unsatisfactory. Furthermore, the comprehensive molecular mechanisms underlying the pathogenesis of T-PLL remain unknown.

The majority of T-PLL cells carry the recurrent chromosome translocations $\mathrm{t}(14 ; 14)(\mathrm{q} 11 ; \mathrm{q} 32) / \mathrm{inv}(14)(\mathrm{q} 11 \mathrm{q} 32)$ or $\mathrm{t}(\mathrm{X} ; 14)(\mathrm{q} 28 ; \mathrm{q} 11)$, which cause the activation of the genes T-cell leukemia/lymphoma 1A $(T C L 1 A)$ or mature T-cell proliferation 1 (6). These genes and their associated pathways are likely to be involved in the progression of T-PLL. Integrated genomic sequencing has proven the importance of mutated DNA or genes in T-PLL (7). Bergmann et al (8) indicated that Janus kinase 3 (JAK3) inhibitors may be an option to treat patients with T-PLL with recurrent activating JAK3 mutations. Genes including TCL1A (9) and SWI/SNF-related matrix-associated actin dependent regulator of chromatin B1 (10) have been demonstrated to be associated with the disease progress of T-PLL. Furthermore, apoptosis has been induced in T-PLL by certain drugs, including bortezomib (11), and by the induction of certain proteins, including p53 (12), which indicates further the potential association between pathways associated with apoptosis and T-PLL. Specific genes and chromosomal loci are likely to be linked with disease progression in T-PLL (13), and identifying the significance of altered genes and pathways is vital to increasing the understanding of T-PLL. However, these genes and pathways have yet to be identified.

To investigate the molecular basis of T-PLL in the present study, a bioinformatics analysis of gene expression profile data (GSE5788) was performed. The differentially expressed genes (DEGs) in T-PLL were identified by comparing the microarray data from 6 T-cell T-PLL blood cell samples with those 
of 8 cluster of differentiation $3(\mathrm{CD} 3)^{+} \mathrm{T}$-cell samples from healthy donors. Gene ontology (GO; http://www.geneontology. org/) function and Kyoto Encyclopedia of Genes and Genomes (KEGG; http://www.genome.jp/kegg/) pathway enrichment analyses were performed, followed by protein-protein interaction (PPI) network and sub-PPI network analyses. The aim was to elucidate the molecular mechanisms of T-PLL, which may aid in the selection of appropriate treatment strategies and the development of novel treatments for T-PLL.

\section{Materials and methods}

Samples. The expression profile dataset GSE5788 (13), which was created using the microarray platform Affymetrix Human Genome U133 Plus 2.0 Array (Santa Clara, CA, USA), was downloaded from the Gene Expression Omnibus (GEO; http://www.ncbi.nlm.nih.gov/geo/). In order to compare the difference between the T-PLL cells and the normal T-cells, a total of 6 T-PLL blood cell samples including GSM135264 (the experimental group), and $8 \mathrm{CD}^{+}$normal donor T-cell samples purified by immunomagnetic separation, including GSM135270 (the control group), were included in GSE5788. The preprocessing of the microarray data, including calculation of the robust multi-array average (14), was performed by using the Affy options in Bioconductor software (15) and the Affy microarray probe annotation file of Brain Array lab (16).

Screening DEGs. An empirical Bayes method based on the Limma package (17) in R software (https://journal.r-project. org/) was used to identify DEGs among the groups. A false discovery rate (FDR) $<0.05$ and log. of fold-change $>1$ were selected as the criteria for the identification of DEGs.

GO and pathway enrichment analysis. GO (18) functional enrichment analysis, including associated cellular component, molecular function (6) and biological process categories, was performed to identify functional enrichment of DEGs. KEGG pathway enrichment (19) was performed to predict the pathways that the previously identified DEGs were associated with. The Database for Annotation, Visualization and Integrated Discovery (DAVID 6.7 Jan. 2010) (20) was used to identify GO categories and significant KEGG pathways with the FDR set as $<0.01$.

Annotation of gene function. The Transcription Factor (TRANSFAC; http://www.gene-regulation.com/pub/ databases.html) database provides information on eukaryotic transcription factors, binding sites, consensus binding sequences and regulated genes. All DEGs were screened based on the TRANSFAC database to identify whether they had a function in transcriptional regulation. Cancer gene databases, including TSGene (a database of tumor suppressor genes) (21) and a tumor-associated gene (TAG) database as described by Chen et al (22), were used to screen for identified cancer-promoting or -inhibiting genes.

PPI network and sub-PPI network construction. The Search Tool for the Retrieval of Interacting Genes (STRING, https://string-db.org/cgi/input.pl) (23) database made associations based on predicted or experimental PPI information.
Table I. Differentially expressed genes in the present study (FDR $<0.05$ and $\log \mathrm{FCl}>1$ ).

\begin{tabular}{lcc}
\hline Type & Transcript count & Gene count \\
\hline Downregulated & 1,249 & 354 \\
Upregulated & 305 & 84 \\
Total & 1,554 & 438 \\
\hline
\end{tabular}

Transcript count refers to the number of differentially expressed transcripts; Gene count refers to the number of DEGs. FDR, false discovery rate; $\mid \log \mathrm{FCl}, \log$ fold-change; DEG, differentially expressed gene.

From the STRING database, protein encoding genes that interacted with specific genes were assembled to construct a PPI network. As the non-DEGs of the network may be associated with multiple DEGs, the result of network analysis may be that the role of non-DEGs is greater compared with that of the DEGs. To study the role of DEGs in PPI and to avoid the interference of non DEGs, the non DEGs associated with 1-2 DEGs were enrolled in the network. Interactions were included when they had a combined score $>0.95$.

The sub-PPI network was additionally investigated using BioNet software (24). The modules were constructed based on the sub-PPI network. A false discovery rate $\mathrm{P} \leq 0.005$ was selected as the threshold for sub-PPI network construction.

\section{Results}

Identification of DEGs. The GSE5788 expression profile data from GEO was investigated to screen for DEGs between the experimental and control groups. A total of 438 DEGs in T-PLL blood sample cells, including 84 upregulated genes and 354 downregulated genes, were revealed (Table I).

GO enrichment analysis. To investigate the function changes in tumor development, GO enrichment analysis of the previously identified DEGs was performed using DAVID. The top 10 up- and downregulated DEGs according to P-value are listed in Table II. The downregulated DEGs were frequently enriched for ontology labels associated with immune function, including immune response (GO:0006955; $\mathrm{P}=3.21 \times 10^{-9}$ ), and tumor progression, including cell death (GO:0008219; $\mathrm{P}=2.37 \times 10^{-7}$ ). Upregulated DEGs were frequently enriched in cell proliferation (GO:0008283; $\mathrm{P}=7.12 \times 10^{-4}$ ) and skin development (GO:0043588; P=9.00x 10-4).

KEGG pathway enrichment analysis. KEGG pathway enrichment analysis using DAVID was performed on the DEGs (Table III). The results revealed that the upregulated DEGs were frequently associated with tumor metastasis pathways, including apoptosis $\left(\mathrm{P}=6.20 \times 10^{-5}\right)$, immune response pathways, including graft-versus-host disease $\left(\mathrm{P}=1.61 \times 10^{-4}\right)$, and immune response or antigen reaction pathways, including Chagas disease (American trypanosomiasis; $\mathrm{P}=5.18 \times 10^{-3}$ ). The downregulated DEGs were enriched in the malaria pathway $\left(\mathrm{P}=3.33 \times 10^{-3}\right)$. 
Table II. Top 10 upregulated and downregulated differentially expressed gene ontologies identified by GO functional enrichment analysis.

A, Downregulated genes

\begin{tabular}{llrr}
\hline GO ID & \multicolumn{1}{c}{ Description } & Gene count & P-value \\
\hline GO:0006955 & Immune response & 58 & $3.21 \times 10^{-9}$ \\
GO:0002376 & Immune system process & 77 & $1.40 \times 10^{-8}$ \\
GO:0008219 & Cell death & 66 & $2.37 \times 10^{-7}$ \\
GO:0016265 & Death & 66 & $2.53 \times 10^{-7}$ \\
GO:0046649 & Lymphocyte activation & 28 & $5.42 \times 10^{-7}$ \\
GO:0042110 & T cell activation & 23 & $5.57 \times 10^{-7}$ \\
GO:0051249 & Regulation of lymphocyte activation & 21 & 18 \\
GO:0030098 & Lymphocyte differentiation & 103 & $1.52 \times 10^{-7}$ \\
GO:0044267 & Cellular protein metabolic process & $1.49 \times 10^{-6}$ \\
\hline
\end{tabular}

B, Upregulated genes

\begin{tabular}{llrr}
\hline GO ID & \multicolumn{1}{c}{ Description } & Gene count & P-value \\
\hline GO:0045321 & Leukocyte activation & 30 & $1.86 \times 10^{-6}$ \\
GO:0008283 & Cell proliferation & 18 & $7.12 \times 10^{-4}$ \\
GO:0043588 & Skin development & 7 & $8.97 \times 10^{-4}$ \\
GO:0009913 & Epidermal cell differentiation & 5 & $1.02 \times 10^{-3}$ \\
GO:0006228 & UTP biosynthetic process & 2 & $1.57 \times 10^{-3}$ \\
GO:0042455 & Ribonucleoside biosynthetic process & 4 & $1.61 \times 10^{-3}$ \\
GO:0046051 & UTP metabolic process & 2 & 3 \\
GO:0006213 & Pyrimidine nucleoside metabolic process & 2 & $2.85 \times 10^{-3}$ \\
GO:1901070 & Guanosine-containing compound biosynthetic process & 4 & $2.15 \times 10^{-3}$ \\
GO:0009163 & Nucleoside biosynthetic process & 4 & $2.21 \times 10^{-3}$ \\
GO:1901659 & Glycosyl compound biosynthetic process & $2.28 \times 10^{-3}$ \\
\hline
\end{tabular}

GO, Gene Ontology; UTP, uridine 5'-triphosphate.

Table III. Top 10 downregulated pathways and a unique upregulated pathway significantly enriched by DEGs in T-cell prolymphocytic leukemia.

\begin{tabular}{llll}
\hline Regulation & \multicolumn{1}{c}{ KEGG pathway } & Gene count & P-value \\
\hline Down & Apoptosis & 9 & $6.20 \times 10^{-5}$ \\
& Graft-versus-host disease & 6 & $1.61 \times 10^{-4}$ \\
& T cell receptor signaling pathway & 9 & $3.30 \times 10^{-4}$ \\
& Allograft rejection & 5 & $8.48 \times 10^{-4}$ \\
& Type I diabetes mellitus & 5 & $1.70 \times 10^{-3}$ \\
& Natural killer cell mediated cytotoxicity & 9 & $1.75 \times 10^{-3}$ \\
& Autoimmune thyroid disease & 5 & $3.96 \times 10^{-3}$ \\
& Antigen processing and presentation & 6 & $4.39 \times 10^{-3}$ \\
& Chagas disease (American trypanosomiasis) & 7 & $5.18 \times 10^{-3}$ \\
Pp & Prion diseases & $4.33 \times 10^{-3}$ & $3.33 \times 10^{-3}$ \\
\hline
\end{tabular}

DEG, differentially expressed gene; KEGG, Kyoto Encyclopedia of Genes and Genomes. 
Table IV. Functional analysis of DEGs.

\begin{tabular}{|c|c|c|c|c|}
\hline \multirow[b]{2}{*}{ Type } & \multicolumn{2}{|r|}{ Downregulated } & \multicolumn{2}{|r|}{ Upregulated } \\
\hline & Count & DEGs & Count & DEGs \\
\hline Transcription factors & 13 & $\begin{array}{l}\text { TBX21, STAT4, STAT3, RORA, } \\
\text { NR3C2, NR3C1, MAF, IRF1,ID2, } \\
\text { GTF2B, GATA3, CDK7, ARNTL }\end{array}$ & 4 & TCF7L2, NME2, KLF4, ENO1 \\
\hline Tumor-associated genes & 27 & & 10 & \\
\hline Oncogenes & 4 & SET, KRAS, GNA13, FYN & 1 & TCL1A \\
\hline Tumor suppressors & 16 & $\begin{array}{l}\text { TGFBR3, TGFBR2, SP100, RARRES3, } \\
\text { PPP } 3 C C, \text { MFHAS1, IRF1, ING3, HOPX, } \\
\text { HECA, HBP1, FHIT, DOK2, CYLD, } \\
\text { CDKN1B, CASP8 }\end{array}$ & 4 & $\begin{array}{l}\text { TCF7L2, RAB25, ESRP1, } \\
C D K 2 A P 1\end{array}$ \\
\hline Others & 7 & $\begin{array}{l}\text { STAT3, PRKCB, MAP } 3 K 5, M A F, \\
\text { EVI2B, EPS15, ATM }\end{array}$ & 5 & KLF4, GSTM1, FES, ENOI, DDRI \\
\hline
\end{tabular}

DEGs, differentially expressed genes.

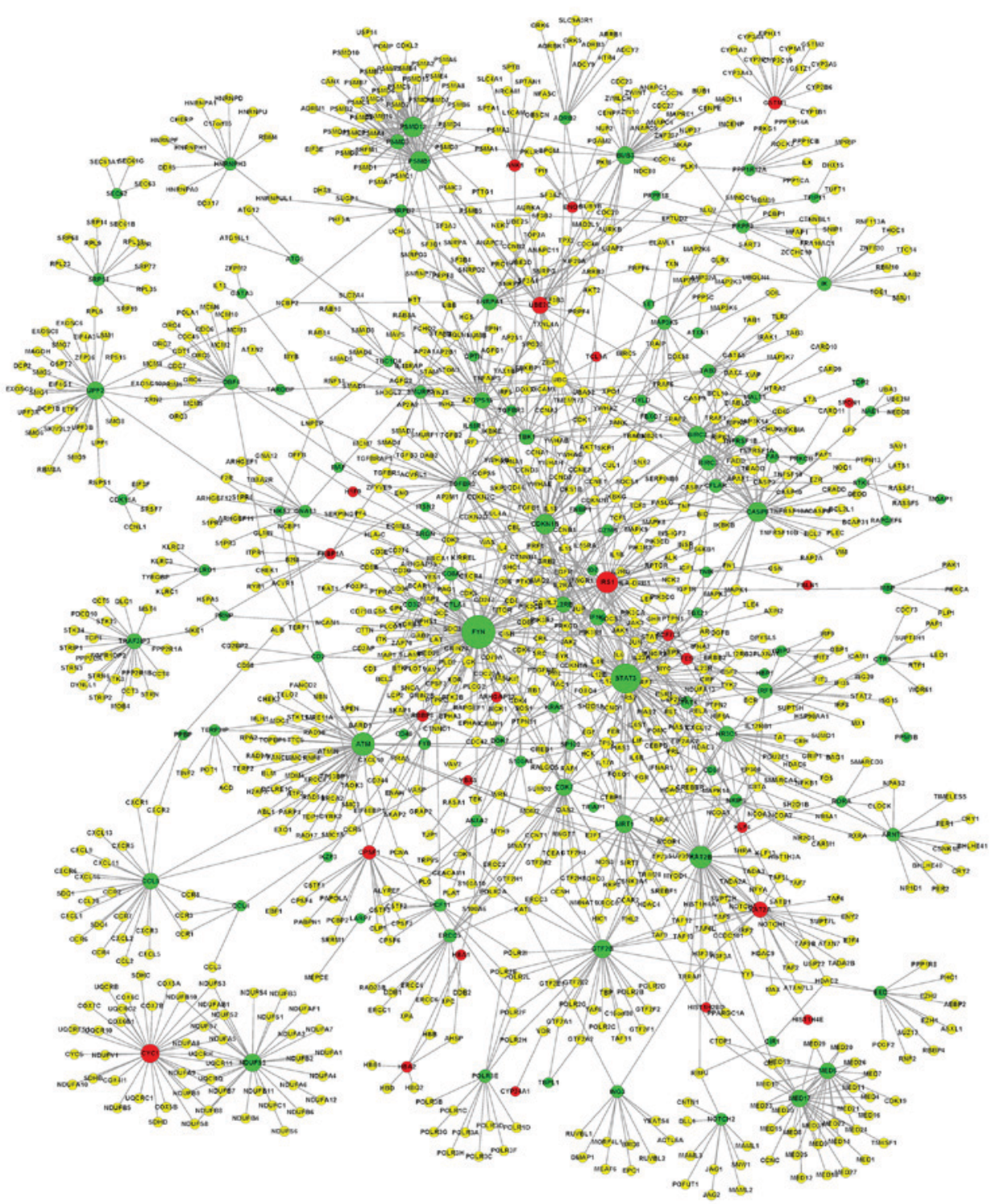

Figure 1. A protein-protein interaction network. The red nodes represent upregulated genes; the green nodes represent the downregulated genes; the yellow nodes represent genes in which expression level was unaltered between T-cell prolymphocytic leukemia and normal T-cells. 


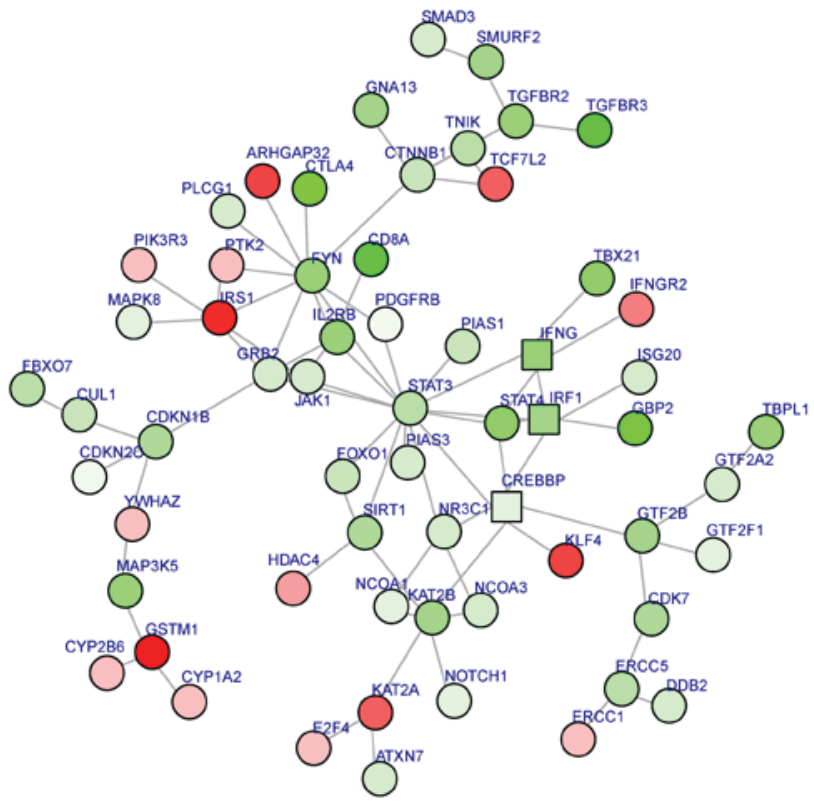

Figure 2. A sub-protein-protein interaction network investigation. The red nodes represent upregulated genes and the green nodes represent downregulated genes in the experimental group (T-cell prolymphocytic leukemia); square nodes represent high importance DEGs and circular nodes represent low importance DEGs in the network. DEGs, differentially expressed genes.

Functional annotation of DEGs. From the T-PLL blood sample microarray data, a total of 13 downregulated [including signal transducer and activator of transcription 3 (STAT3)] and 4 upregulated [including transcription factor 7 like 2 (TCF 7L2)] transcription factors, as well as 27 downregulated (including $F Y N$ ) and 10 upregulated (including TCL1A) TAGs were revealed to be associated with T-PLL (Table IV).

Using the Disease Ontology database (25), the downregulated genes, including $A R L 6 I P 5, A T M, C C L 4, C C L 5$, CDKN1B, CFLAR, FAS, GNLY, IL2RB, KAT2B, MAP3K5, SET and TRIM22, were revealed to be associated with leukemia and chronic lymphocytic leukemia.

PPI module investigation and pathway regulation analysis. A PPI network of DEGs associated with T-PLL was constructed based on the STRING database (Fig. 1). A total of 10 nodes with the highest degree were selected, including $F Y N$ (degree, 90), STAT3 (degree, 76), ATM (degree, 51), KAT2B (degree, 48), IRSI (insulin receptor substrate-1; degree, 45), PSMD12 (degree, 41), PSMB1 (degree, 40), CDKN1B (degree, 40), CASP8 (degree, 38) and $C Y C l$ (degree, 34).

A sub-PPI network was constructed based on the aforementioned PPI network. BioNet software was used to analyze the sub-PPI network. A total of 61 gene nodes, including STAT3 (the most significantly downregulated gene in the sub-PPI network, degree, 14) and IRS1 (the most significantly upregulated gene in the sub-PPI network, degree, 6), were included in the sub-PPI network (Fig. 2).

The KEGG pathway analysis was performed based on the DEGs in the sub-PPI network. As presented in Table V, the sub-network of STAT3 is involved in growth signal pathways [including the JAK-STAT signaling pathway $\left(\mathrm{P}=3.0 \times 10^{-8}\right)$ ], cell differentiation pathways [including osteoclast differentiation
Table V. The top 10 most KEGG-enriched pathways for the network module.

\begin{tabular}{lcc}
\hline KEGG pathway & Gene count & P-value \\
\hline Pathways in cancer & 17 & $2.65 \times 10^{-10}$ \\
JAK-STAT signaling pathway & 11 & $2.97 \times 10^{-8}$ \\
Prostate cancer & 8 & $4.67 \times 10^{-7}$ \\
TGF- $\beta$ signaling pathway & 7 & $4.37 \times 10^{-6}$ \\
Cell cycle & 8 & $5.88 \times 10^{-6}$ \\
Osteoclast differentiation & 8 & $7.45 \times 10^{-6}$ \\
Colorectal cancer & 6 & $9.43 \times 10^{-6}$ \\
Hepatitis C & 8 & $1.05 \times 10^{-5}$ \\
Pancreatic cancer & 6 & $1.91 \times 10^{-5}$ \\
T cell receptor signaling pathway & 7 & $2.32 \times 10^{-5}$ \\
\hline
\end{tabular}

KEGG, Kyoto Encyclopedia of Genes and Genomes; TGF, transcription growth factor.

$\left.\left(\mathrm{P}=7.5 \times 10^{-6}\right)\right]$, cancer-associated pathways [including prostate cancer $\left.\left(\mathrm{P}=4.7 \times 10^{-7}\right)\right]$, and viral disease-associated pathways [including hepatitis $\mathrm{C}\left(\mathrm{P}=1.1 \times 10^{-5}\right)$ ].

\section{Discussion}

T-PLL is a rare, aggressive T-cell leukemia, which has not been well characterized, particularly in terms of its molecular mechanisms. In the present study, the molecular pathogenesis of T-PLL was investigated based on a comprehensive bioinformatics analysis. The results identified 84 upregulated and 354 downregulated genes in T-PLL sample microarrays. These DEGs were associated with various functions including cell death, and various pathways, including apoptosis. A total of 17 dysregulated transcription factors and 37 dysregulated TAG were revealed based on functional analysis of DEGs. A PPI network analysis identified a total of 61 genes. The most significantly downregulated gene, STAT3 (degree, 14), and upregulated gene, IRS1 (degree, 6), may have significant associations with the pathogenesis and progression of T-PLL.

The dysregulation of specific genes, including transcription factors, and associated pathways is commonly associated with increased tumor cell proliferation, based on previous bioinformatics analyses (26-28). These genes and pathways perform important roles and are likely to be significant in the development of cancer (29). STAT3 is activated in various types of cancer, including gliomas and breast cancer $(30,31)$. The STAT3 signaling pathway, including the upstream JAK signal transducer, has been reported to participate in the development of various cancer types $(32,33)$.

Previous studies indicate that JAK2-STAT3 signaling is involved in the production of hepatic thrombopoietin (34) and the growth of hormone refractory prostate cancer cells (35). In the present study, the downregulated STAT3 was the core node of the sub-PPI network, and the DEGs connected to STAT3 were involved in pathways including JAK-STAT signaling. This result confirms that STAT3 and the JAK2-STAT3 pathway are associated with the progression of T-PLL. 
Another gene identified to be significant was IRSI, a critical component of insulin signaling, which is also involved in cell proliferation and cancer development (36). IRS1 is associated with the progression of tumors, including lung cancer (37) and colorectal cancer (38). The significant upregulation of IRSI in the present study indicated the close association of IRS1 with T-PLL, which was in accordance with the function of IRSI in cancer identified in previous studies. The expression levels of various genes, including STAT3 and IRS1, were significantly altered in the tumor compared with the controls, implying they may be used as novel biomarkers for establishing a prognosis in T-PLL.

Novel drugs targeting specific pathways can be developed based on an understanding of the pathogenesis of T-PLL (5). KEGG pathway analysis in the present study revealed that apoptosis and T-cell receptor signaling were included among the enriched pathways identified. The majority of these outstanding pathways were enriched among the downregulated genes, indicating that the downregulation of genes in these pathways may act to inhibit T-cell activation, promoting disease progression. However, additional investigations are required to improve the understanding of the complex interaction of these dysregulated genes and associated pathways.

In conclusion, the mechanism of T-PLL was observed to be complicated. Various cell functions, including cell death, and pathways, including apoptosis, may be involved in the process. Identified candidate genes, including STAT3 and IRSI, may be targets for the additional study of T-PLL.

\section{Acknowledgements}

Not applicable.

\section{Funding}

This work was supported by the Lateral projects of Jilin University (Grant No. 3R216X133430 and 3R216X123430).

\section{Availability of data and materials}

The expression profile dataset GSE5788, was downloaded from the Gene Expression Omnibus (GEO; http://www.ncbi. nlm.nih.gov/geo/).

\section{Authors' contributions}

ZS and HL designed the bioinformatics pipeline. JY, HS, KC, JZ and QJ performed the bioinformatics analysis. ZS and JY prepared the manuscript.

\section{Ethics approval and consent to participate}

Not applicable.

\section{Consent for publication}

Not applicable.

\section{Competing interests}

The authors declare that they have no competing interests.

\section{References}

1. Herling M, Khoury JD, Washington LT, Duvic M, Keating MJ and Jones D: A systematic approach to diagnosis of mature T-cell leukemias reveals heterogeneity among WHO categories. Blood 104: 328-335, 2004.

2. Matutes E, Brito-Babapulle V, Swansbury J, Ellis J, Morilla R, Dearden C, Sempere A and Catovsky D: Clinical and laboratory features of 78 cases of T-prolymphocytic leukemia. Blood 78: 3269-3274, 1991

3. Soulier J, Pierron G, Vecchione D, Garand R, Brizard F, Sigaux F, Stern MH and Aurias A: A complex pattern of recurrent chromosomal losses and gains in T-cell prolymphocytic leukemia. Genes Chromosomes Cancer 31: 248-254, 2001.

4. Vivekanandarajah A, Atallah JP and Gupta S: T-cell prolymphocytic leukaemia (T-PLL): A rare disease with a grave prognosis. BMJ Case Rep 2013: pii: bcr2013009808, 2013.

5. K A and D C: T-cell prolymphocytic leukemia. Clin Lymph Myelom 9 (Suppl 3): S239-S243, 2009.

6. Brito-Babapulle V, Pomfret M, Matutes E and Catovsky D: Cytogenetic studies on prolymphocytic leukemia. II. T cell prolymphocytic leukemia. Blood 70: 926-931, 1987.

7. Kiel MJ, Velusamy T, Rolland D, Sahasrabuddhe AA, Chung F, Bailey NG, Schrader A, Li B, Li JZ, Ozel AB, et al: Integrated genomic sequencing reveals mutational landscape of T-cell prolymphocytic leukemia. Blood 124: 1460-1472, 2014.

8. Bergmann AK, Schneppenheim S, Seifert M, Betts MJ, Haake A, Lopez C, Maria Murga Penas E, Vater I, Jayne S, Dyer MJ, et al: Recurrent mutation of JAK3 in T-cell prolymphocytic leukemia. Genes Chromosomes Cancer 53: 309-316, 2014.

9. Yokohama A, Saitoh A, Nakahashi H, Mitsui T, Koiso H, Kim Y, Uchiumi H, Saitoh T, Handa H, Jimbo T, et al: TCL1A gene involvement in T-cell prolymphocytic leukemia in Japanese patients. Int J Hematol 95: 77-85, 2012.

10. Bug S, Dürig J, Oyen F, Klein-Hitpass L, Martin-Subero JI, Harder L, Baudis M, Arnold N, Kordes U, Dührsen U, et al: Recurrent loss, but lack of mutations, of the SMARCB1 tumor suppressor gene in T-cell prolymphocytic leukemia with TCL1A-TCRAD juxtaposition. Cancer Genet Cytogenet 192: 44-47, 2009.

11. Ozpuyan F, Meyer P, Ni H, Al-Masri H and Alkan S: Bortezomib induces apoptosis in T-cell prolymphocytic leukemia (T-PLL). Leuk Lymphoma 48: 2247-2250, 2007.

12. Wang J, Hasui K, Utsunomiya A, Jia X, Matsuyama T and Murata F: Association of high proliferation in adult T-cell leukemia cells with apoptosis, and expression of $\mathrm{p} 53$ protein in acute type ATL. J Clin Exp Hematop 48: 1-10, 2008.

13. Dürig J, Bug S, Klein-Hitpass L, Boes T, Jöns T, Martin-Subero JI, Harder L, Baudis M, Dührsen U and Siebert R: Combined single nucleotide polymorphism-based genomic mapping and global gene expression profiling identifies novel chromosomal imbalances, mechanisms and candidate genes important in the pathogenesis of T-cell prolymphocytic leukemia with inv(14) (q11q32). Leukemia 21: 2153-2163, 2007.

14. Irizarry RA, Hobbs B, Collin F, Beazer-Barclay YD, Antonellis KJ, Scherf U and Speed TP: Exploration, normalization and summaries of high density oligonucleotide array probe level data. Biostatistics 4: 249-264, 2003.

15. Gautier L, Cope L, Bolstad BM and Irizarry RA: Affy-analysis of Affymetrix GeneChip data at the probe level. Bioinformatics 20: 307-315, 2004.

16. Wang P, Ding F, Chiang H, Thompson RC, Watson SJ and Meng F: ProbeMatchDB-a web database for finding equivalent probes across microarray platforms and species. Bioinformatics 18 : 488-489, 2002.

17. Smyth GK: Linear models and empirical bayes methods for assessing differential expression in microarray experiments. Stat Appl Genet Mol Biol 3: Article3, 2004.

18. Ashburner M, Ball CA, Blake JA, Botstein D, Butler H, Cherry JM, Davis AP, Dolinski K, Dwight SS, Eppig JT, et al: Gene ontology: Tool for the unification of biology. The Gene Ontology Consortium. Nat Genet 25: 25-29, 2000.

19. Kanehisa M and Goto S: KEGG: Kyoto encyclopedia of genes and genomes. Nucleic Acids Res 28: 27-30, 2000.

20. Huang da W, Sherman BT and Lempicki RA: Systematic and integrative analysis of large gene lists using DAVID bioinformatics resources. Nat Protoc 4: 44-57, 2009.

21. Zhao M, Sun J and Zhao Z: TSGene: A web resource for tumor suppressor genes. Nucleic Acids Res 41 (Database Issue): D970-D976, 2013. 
22. Chen JS, Hung WS, Chan HH, Tsai SJ and Sun HS: In silico identification of oncogenic potential of fyn-related kinase in hepatocellular carcinoma. Bioinformatics 29: 420-427, 2013.

23. Franceschini A, Szklarczyk D, Frankild S, Kuhn M, Simonovic M, Roth A, Lin J, Minguez P, Bork P, von Mering C and Jensen LJ: STRING v9.1: Protein-protein interaction networks, with increased coverage and integration. Nucleic Acids Res 41 (Database Issue): D808-D815, 2013.

24. Beisser D, Klau GW, Dandekar T, Müller T and Dittrich MT: BioNet: An R-Package for the functional analysis of biological networks. Bioinformatics 26: 1129-1130, 2010

25. Kibbe WA,ArzeC,Felix V,Mitraka E, BoltonE,Fu G,MungallCJ, Binder JX, Malone J, Vasant D, et al: Disease Ontology 2015 update: An expanded and updated database of human diseases for linking biomedical knowledge through disease data. Nucleic Acids Res 43 (Database Issue): D1071-D1078, 2015.

26. Liu YJ, Zhu Y, Yuan HX, Zhang JP, Guo JM and Lin ZM: Overexpression of HOXC11 homeobox gene in clear cell renal cell carcinoma induces cellular proliferation and is associated with poor prognosis. Tumour Biol 36: 2821-2829, 2015.

27. Li W, Li K, Zhao L and Zou H: Bioinformatics analysis reveals disturbance mechanism of MAPK signaling pathway and cell cycle in Glioblastoma multiforme. Gene 547: 346-350, 2014.

28. Verstrepen L and Beyaert R: Receptor proximal kinases in $\mathrm{NF}-\kappa \mathrm{B}$ signaling as potential therapeutic targets in cancer and inflammation. Biochem Pharmacol 92: 519-529, 2014.

29. Liu J, Li J, Li H, Li A, Liu B and Han L: A comprehensive analysis of candidate genes and pathways in pancreatic cancer. Tumour Biol 36: 1849-1857, 2015.

30. Kim HS, Li A, Ahn S, Song $\mathrm{H}$ and Zhang W: Inositol Polyphosphate-5-Phosphatase F (INPP5F) inhibits STAT3 activity and suppresses gliomas tumorigenicity. Sci Rep 4: 7330, 2014.

31. Tell RW and Horvath CM: Bioinformatic analysis reveals a pattern of STAT3-associated gene expression specific to basal-like breast cancers in human tumors. Proc Natl Acad Sci USA 111: 12787-12792, 2014.
32. Santillán-Benítez JG, Mendieta-Zerón H, Gómez-Oliván LM, Ordóñez Quiroz A, Torres-Juárez JJ and González-Bañales JM: JAK2, STAT3 and SOCS3 gene expression in women with and without breast cancer. Gene 547: 70-76, 2014.

33. Gao SM, Chen CQ, Wang LY, Hong LL, Wu JB, Dong PH and Yu FJ: Histone deacetylases inhibitor sodium butyrate inhibits JAK2/STAT signaling through upregulation of SOCS1 and SOCS3 mediated by HDAC8 inhibition in myeloproliferative neoplasms. Exp Hematol 41: 261-270.e4, 2013.

34. Grozovsky R, Begonja AJ, Liu K, Visner G, Hartwig JH, Falet H and Hoffmeister KM: The Ashwell-Morell receptor regulates hepatic thrombopoietin production via JAK2-STAT3 signaling. Nat Med 21: 47-54, 2015.

35. Jorvig JE and Chakraborty A: Zerumbone inhibits growth of hormone refractory prostate cancer cells by inhibiting JAK2/STAT3 pathway and increases paclitaxel sensitivity. Anticancer Drugs 26: 160-166, 2015.

36. Ozoe A, Sone M, Fukushima T, Kataoka N, Arai T, Chida K, Asano T, Hakuno F and Takahashi S: Insulin receptor substrate-1 (IRS-1) forms a ribonucleoprotein complex associated with polysomes. FEBS Lett 587: 2319-2324, 2013.

37. Hu Y, He K, Wang D, Yuan X, Liu Y, Ji H and Song J: TMEPAI regulates EMT in lung cancer cells by modulating the ROS and IRS-1 signaling pathways. Carcinogenesis 34: 1764-1772, 2013

38. Li P, Wang L, Liu L, Jiang H, Ma C and Hao T: Association between IRS-1 Gly972Arg polymorphism and colorectal cancer risk. Tumour Biol 35: 6581-6585, 2014.

(7) $\Theta$ This work is licensed under a Creative Common International (CC BY-NC-ND 4.0) License. 